\title{
Density of test bodies and its effect on burning rate of fire-retardant treated samples
}

\author{
Linda Makovická Osvaldová \\ University of Žilina in Žilina, Faculty of Security Engineering, Žilina, Slovakia
}

\begin{abstract}
The thesis focuses on the effect of wood density on the results of fire tests. Wood (spruce wood in particular) is one of the most commonly used materials in many sectors - building constructions, furniture manufacture etc. With such a broad application, its fire technical properties must not be neglected. Fire retardants should be therefore used to prevent the fire from occurring and spreading.
\end{abstract}

\section{Introduction}

This physical property affects all technological processes as well as mechanical properties [4,1]. Therefore it has a direct impact on the production of components and parts for wooden constructions.

Wood density has an impact on other aspects of wood evaluation and its properties i.e. evaluation of firetechnical proprieties and retarding adjustments. Regardless of evaluation methodology (standard test methods or conical calorimeter) if the change in weight or thermal properties are used as the evaluation criteria, density may affect the final results of measurements. Test methods almost always specify the parameters for wood moisture, air conditioning, thickness or quality of working, however, the parameters for density (limit values at least) are missing. This article aims to identify the effect of density of spruce wood test specimens on the results of the experiments.

\section{Material}

Spruce wood (Picea abies (L) Karst Records.) is the second most widespread type of coniferous wood in Slovakia and the most important one from the economic point of view.

Different parts of tree (root, trunk and tree crown) have different functions and usability.

Chemistry in a live tree indicates chemical composition of necrotic cells (cell walls and lumens - inter-cellular spaces). From chemical viewpoint, wood is a complicated complex of heterogeneous bio-polymers $(90-97 \%)$ socalled major components accompanied by smaller quantities of accompanying components (3 - $10 \%)$. Main components of wood are represented by saccharide share $(65-75 \%)$ - composed of cellulose $(40-50 \%)$ and hemicelluloses - plus aromatic share formed by lignin $(15-35 \%)$. Accompanying (accessory) components of wood are formed by organic substances - monomers and polymers - as well as by inorganic ones. In addition to organic substances, which are an essential part of wood, wood also contains mineral substances creating ash during combustion.

Cellulose is a high molecular polysaccharide consisting of B-D- anhydro-derivate of beta-D glucopyranose $(1 \rightarrow 4)$.[1] For wood, average polymerization degree of natural cellulose evaluated viscosymetrically is 4000 up to 5500. One part of cellulose chains remains in amorphous arrangement while continuous transition between crystalline and amorphous share can be observed. Cellulose chains are maintained by hydrogen bonds between hydroxy groups of neighboring cellulosic chains.

Hemicelluloses are composed of heteropolysaccharides with lower polymerization degree (mostly 100 to 200). The main components are pentose and hexose: Lrahmnose, L- fructose, L- arabinose, D- xylose, Dmannose, D-glucose and D- galactoze. Hemicelluloses are amorphous and contain neutral or acidic cross-linked fibres. Coniferous plants contain glucomannans - the main hemicellulose component. Depending on the type of plant, their amount ranges from 20 to $30 \%$.[2] Lignin is a benzenoid component of some plants containing benzene core with propane chain, phenolic hydroxyl groups and forms a three-dimensional macromolecules.

Coniferous wood after thermal degradation is present by three changes - changes in chemical composition, in anatomical structure and in physical characteristic.

There are materials entering the reaction with oxygen (e.g. metals) or which, in the first stage of heating, change their chemical composition, or the composition of their core building elements, produce flammable gases and begin to burn. Wood belongs to this category too. Main components of wood degrade at different temperatures affecting the whole process of combustion. Hemicelluloses will decompose under temperatures 
ranging between $170-240{ }^{\circ} \mathrm{C}$. They are the least resistant to thermal decomposition. By regulating wood heating, we may achieve reduction of hemicelluloses without reducing its strength, resulting in an increase in dimensional stability of wood. [3]

Cellulose is more resistant to thermal stress than hemicelluloses. Decomposition rate of cellulose is moderate up to $250{ }^{\circ} \mathrm{C}$. Intensive decomposition of cellulose is observed at the temperatures of $250-300{ }^{\circ} \mathrm{C}$. It leads to disruption of links in the main chain according to radical mechanism, the tail link of cellulose turns into levoglucosan.[3]

Lignin is the most resistant to thermal decomposition. Process of the thermal decomposition of lignin is divided into two stages. In the first stage, labile ether alkyl links decompose at the temperatures of $300-320{ }^{\circ} \mathrm{C}$. The second stage is an active decomposition of lignin at the temperatures of $350-390{ }^{\circ} \mathrm{C}$. The structure of macromolecules is disrupted and volatile products are being released. Formation of volatile products is lower than with cellulose. Decomposition rate is slowing down by increasing the temperature and is followed by accumulation of condensing aromatic structures in the solid phase.[4]

Wood exposed to temperatures higher than $288{ }^{\circ} \mathrm{C}$ (temperature generally assumed as charring temperature) can be divided into five degradation zones.[4] The variety of the decomposition of main wood components and characteristic reactions in different temperature intervals lead to differentiation of burning process into several stages.

Anatomical structure of wood - individual cell elements affects the process of combustion, its first phase ignition - in particular. It results mainly from chemical composition and geometric shape of cellular elements, their size and their number.

In addition to chemical composition, physical properties of wood and wood-based materials significantly influence the course of combustion. Each physical property has an impact on burning, though not to the same degree. [5]

Surface of the material (its quality) is another physical characteristic, which significantly affects combustion. Wood, capillary-porous material, is rough on the surface. The roughness depends on working as well as on anatomical structure of wood. Besides roughness, surface quality also depends on anatomical defects, defects resulting from working, mechanical damage, dirt and etc., changing the surface quality. Surface quality mainly affects thermal conductivity $\alpha$. Smooth and high-quality surface reflects energy of radiation source and flame source thus making it more difficult for wood to be ignited compared to rough surface under the same test conditions.

From the point of view of quality, working is as important as the color of wood. Natural color of wood (including tropical woody plants) comprises the entire color range. Thermal degradation causes color changes. Light color of wood gradually darkens and finally turns black - charred layer. Intensity of darkening depends on heat rate and temperature - several authors treated the subject. Color change is brought about by polysaccharide degradation (hemicelluloses and cellulose). [5]
Test specimens made from spruce wood are sorted out according to their density. Out of 450 specimens, 60 pieces have been divided into three density groups (see Table 1).

\subsection{Selection criteria}

\subsubsection{Density}

$$
\rho=m / V \quad\left(k g / m^{3}\right)
$$

where:

$\rho-\operatorname{density~}\left(\mathrm{kg} / \mathrm{m}^{3}\right)$

$m-$ weight $(\mathrm{kg})$

$V$ - volume $\left(\mathrm{m}^{3}\right)$

According to the following formula, density of the selected samples has been calculated. Samples have been then arranged from the ones of lowest to highest density. All samples have been then divided into three categories. Samples of the lowest densities with the average value of $381.5 \mathrm{~kg} / \mathrm{m}^{3}$ have been included into low density group. Samples of medium values have been included into medium density category. The average values of all samples were $437.87 \mathrm{~kg} / \mathrm{m}^{3}$. Samples with the highest values have been included into the category of high density - their average density value was $495.62 \mathrm{~kg} / \mathrm{m}^{3}$. Each category was divided into two groups - fireretardant treated samples and untreated samples. Their average values are shown in table 1 .

Table 1. Average densities of samples.

\begin{tabular}{|c|c|c|}
\hline Density $\mathbf{( k g / \mathbf { m } ^ { \mathbf { 3 } } )}$ & $\begin{array}{c}\text { Average density } \\
\text { of untreated } \\
\text { samples }\end{array}$ & $\begin{array}{c}\text { Average density } \\
\text { of fire- } \\
\text { retardant } \\
\text { treated samples }\end{array}$ \\
\hline Low density & 378.0 & 385.0 \\
\hline Medium density & 438.4 & 437.4 \\
\hline High density & 492.4 & 498.9 \\
\hline
\end{tabular}

\subsubsection{Fire retardant}

Fire retardant called Ohňostop was randomly selected for the experiment. The retardant will not be described or characterized in details. The goal of the experiment was not to evaluate the retardant. Our goal is to find out whether the density of test bodies has an effect on the overall result even with fire retardant treated samples.

\subsubsection{Thermal load}

The method of heat load application is based on standard test method where weight loss is the main assessment criterion. Test specimens of $200 \times 100 \times 10$ $\mathrm{mm}$ (10 mm - thickness) have been exposed to the flame 
of a gas burner for 10 minutes. The flame has been directed at the middle of the test specimen which formed $45^{\circ}$ angle with the horizontal plane. Perpendicular distance of the center of the sample and the mouth of the burner was $90 \mathrm{~mm}$. Flame is $100 \mathrm{~mm}$ long.

\subsection{Evaluation criteria}

Relative burning rate has been determined according to the following relations (2) (3):

$$
v_{r}=\left|\frac{\partial \delta_{m}}{\partial \tau}\right| \quad(\% / \mathrm{s})
$$

or numerically

$$
v_{r}=\frac{\left|\delta_{m}(\tau)-\delta_{m}(\tau+\Delta \tau)\right|}{\Delta \tau}
$$

where:

$v_{r}$ - relative burning rate $(\% / \mathrm{s})$,

$\delta_{m}(\tau)$ - relative weight loss in certain time $(\tau)(\%)$,

$\delta_{m}(\tau+\Delta \tau)$ - relative weight loss in certain time $(\tau+\Delta \tau)$ $(\%)$,

$\Delta \tau$ - time interval at which weights are being subtracted (s).

\section{Evaluation}

Labels were as follows: NNX for untreated samples and RNX for fire-retardant treated samples of low density; NSX for untreated samples and RSX for fire- retardant treated samples of medium density; NVX for untreated samples of high density samples and RVX for the samples treated with a flame retardant.

Maximum relative burning rate of $0.0958 \mathrm{~s} / \%$ for NNX samples was achieved in 60th second. Maximum relative burning rate of $0.0476 \mathrm{~s} / \%$ for RNX samples was recorded in 80th second. These values of relative burning rate of treated and untreated samples of low density show significant differences between the two groups proving the importance of fire-retardant application. These differences can be seen from figure 1 .

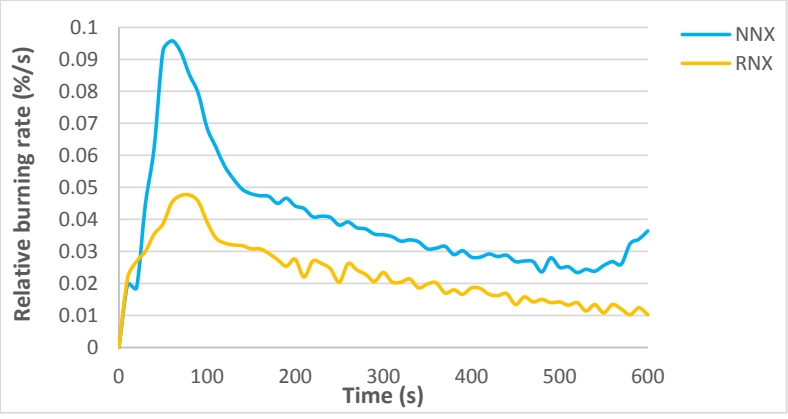

Figure 1. Average relative burning rate of low density samples.
Maximum burning rate for RSX samples was $0.0496 \mathrm{~s} / \%$ in 60th second. We observe greater differences between the values of relative burning rate of medium density samples treated with fire retardant and untreated ones compared with low-density samples. These differences are shown in figure 2 .

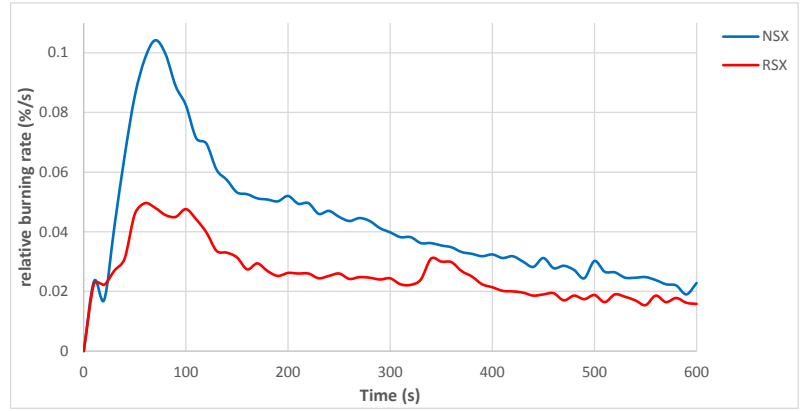

Figure 2. Average relative burning rate of medium density samples.

Maximum relative burning rate for NVX samples was $0.0862 \mathrm{~s} / \%$ in 80th second. Maximum relative burning rate for RVX samples was $0.0512 \mathrm{~s} / \%$ in 70 th second. The smallest difference can be observed between these values - weight loss and relative burning rate of treated and untreated samples of high density - compared to samples of low and medium density. These differences are shown in figure 3 .

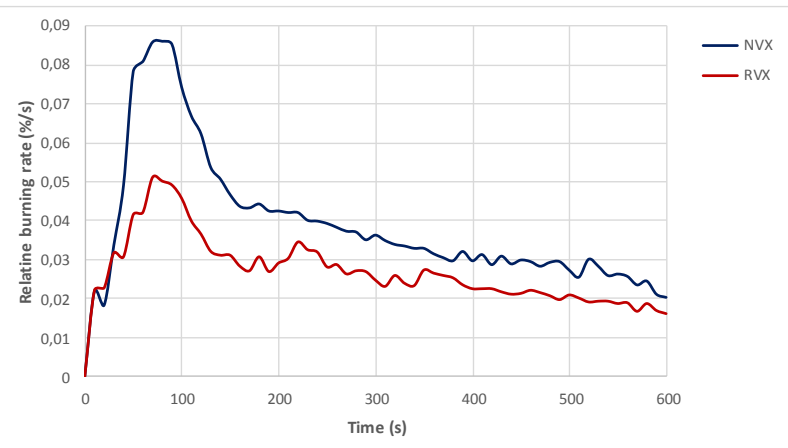

Figure 3. Average relative burning rate of samples of high density.

\subsection{Overall evaluation of laboratory tests}

Overall evaluation of laboratory tests has been done using all the average values, i.e. average values of untreated samples of low density (NNX), fire retardant treated samples of low density (RNX), untreated medium density samples (NSX), medium density samples treated with a fire retardant (RSX), untreated high density samples (NVX) and high density samples treated with a fire retardant (RVX).

Based on the values shown in the table 2 and fig. 4, following facts can be concluded:

- Fire retardant played an important role since the untreated samples of low, medium and high density 
suffered greater weight loss than the samples of low, medium and high density treated with a fire retardant.

- The greatest difference between the treated and untreated samples occurred in the case of medium density samples since these samples have absorbed the largest quantity of fire retardant.

- On the other hand, the smallest difference between the treated and untreated samples occurred in case of high density samples since they absorbed the lowest quantity of fire retardant.

- The greatest weight loss was recorded for untreated samples of low density.

- The lowest weight loss was observed in the case of samples of medium density treated with a fire retardant.

- Each group of untreated samples has achieved higher maximum relative burning rate than the untreated ones.

- The highest relative burning rate has been observed with the untreated samples of medium density since the value was achieved 10 seconds later than with the untreated samples of low density that achieved the second highest relative burning rate.

- The lowest relative burning rate has been recorded for fire-retardant treated samples of low density.

Table 2. Evaluation of laboratory tests.

\begin{tabular}{|c|c|}
\hline Density - label & $\begin{array}{c}\text { Relative burning rate } \\
\mathbf{( \% / s )}\end{array}$ \\
\hline NNX & 0.0958 \\
\hline RNX & 0.0476 \\
\hline NSX & 0.01042 \\
\hline RSX & 0.0946 \\
\hline NVX & 0.0862 \\
\hline RVX & 0.0512 \\
\hline
\end{tabular}

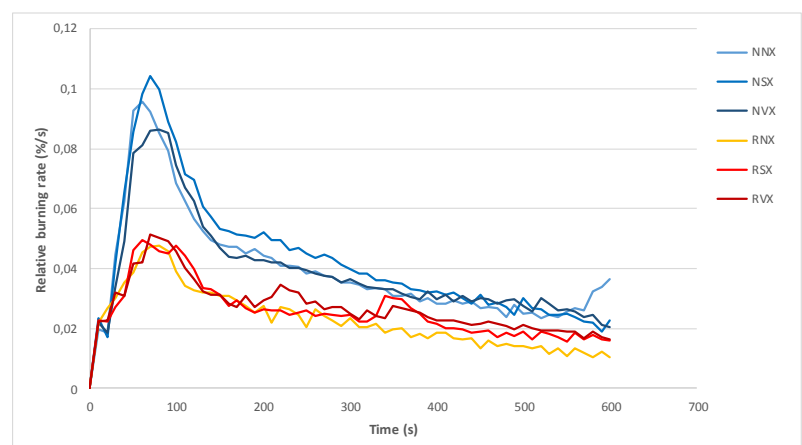

Figure 4. Relative burning rate of average values (all samples).

\section{Conclusion}

In conclusion - density of wooden test bodies has a direct effect on the results of measurements of retarding effect of fire retardants. The experiment monitored the evaluation criteria related to weight or its change during the evaluation of fire retardants. Experiment confirmed that the more retardant is applied, the lesser is the effect of density on test results, and however, this aspect cannot be neglected. Moreover, if quality selection of test specimens - according to their density before the treatment/experiment - is not carried out, a great variability of results in the given group can occur. The auxiliary statistical criteria may affect the overall evaluation of the given fire retardant. We should point out that density will have an effect on the evaluation criteria which aim to track the changes in temperature or the effect of heat flow $[6,7,8]$.

\section{Acknowledge}

This work was supported by the Slovak Grand agency VEGA. (Project 1/022/16|6| Fire safe insulation systems based on natural materials.).

\section{References}

1. Osvald A, Makovická Osvaldová L, Kačíková D. Coniferous wood - reaction on fire. In: Wood and Fire Safety; 13. - 16. May 2012; The High Tatras Štrbské Pleso, Slovakia. 2012. p. 203-210.

2. Bubeníková T, Kačík F, Kačíková D. Characteristic of lignins at low temperature degradation of spruce wood. In: Proccedings Wood and Fire Safety; 2004; Štrbské Pleso, Slovakia. Zvolen, Slovakia: Technická Univerzita; 2004. p. 25-30.

3. Hawley LF. Combustion of wood. Wood chemistry. 1952;II:548.

4. White RH, Nordheim EV. Charring Rate of Wood for ASTM E 119 Exposure. Fire technology. 1992;28(1):5-30.

5. Martinka, J., Chrebet, T., 2014 a: Activation energy of Teak and Oak wood spontaneous ignition. In: Advanced Materials Research, Switzerland, vol. 1001, 2014, pp. 262-266.F. De Lillo, F. Cecconi, G. Lacorata, A. Vulpiani, EPL, 84 (2008)

6. XU Qiang, QUE Xinggui, CAO Liying, JIANG Yong, JIN Cong, 2010: Study of heat flux distribution of wood crib fires: bench scale tests, Thermal Science, 2010, Vol. 14, No.1,pp 283-290

7. Tsuomis, G., 1991: Science and technology of wood; Structure,properties, utilization. NewYork :Chapman and Hall, p. 494. ISBN 0-412-07851-1

8. Kal'avská L'ubomíra : [Diploma thesis].University of Žilina. Faculty of Security Engineering;Department of fire engineering, study programme: Rescue services. 2016, 62 pages 